TTR

Traduction, terminologie, rédaction

\title{
Traduction et éditorialisation des récits sur la banlieue : paratexte iconique et transfert culturel
}

\section{Béatrice Turpin}

Volume 31, numéro 1, 1er semestre 2018

Traduire la banlieue : problématiques, enjeux, perspectives Translating the Banlieue: Issues, Challenges, Perspectives

URI : https://id.erudit.org/iderudit/1062545ar

DOI : https://doi.org/10.7202/1062545ar

Aller au sommaire du numéro

\section{Éditeur(s)}

Association canadienne de traductologie

ISSN

0835-8443 (imprimé)

1708-2188 (numérique)

Découvrir la revue

Citer cet article

Turpin, B. (2018). Traduction et éditorialisation des récits sur la banlieue : paratexte iconique et transfert culturel. TTR, 31(1), 23-46. https://doi.org/10.7202/1062545ar

\section{Résumé de l'article}

Une première de couverture est un discours composite qui résulte de multiples choix éditoriaux. Elle doit refléter l'identité de la maison d'édition dont elle est la vitrine ainsi que celle de la collection qui ouvre des pistes de lecture. Elle doit également attirer le lecteur et lui donner une idée du contenu de l'ouvrage. Le présent article étudie l'éditorialisation de quelques récits sur la banlieue représentatifs de la littérature urbaine de la fin du $\mathrm{XX}^{\mathrm{e}}$ siècle et du début du XXI ${ }^{\mathrm{e}}$ siècle. L'analyse des publications françaises montre la prégnance d'un point de vue réaliste, avec une couverture qui peut être considérée comme une image arrêtée d'un scénario (souvent reprise d'un film qui a vu le succès du livre). Celle-ci tend par ailleurs à personnaliser l'ouvrage, avec la représentation en plan rapproché d'un narrateur/personnage. Les éditions traduites tendent pour leur part à élargir le champ du regard en contextualisant le cadre historique ou spatial du récit ou, au contraire, effacent celui-ci. Le motif mis en scène, énigmatique, devient symbole ouvert sur de multiples interprétations. L'ouvrage, par là même, sort du cadre de la littérature dite " de banlieue ». Ces deux tendances, emblématiques de choix éditoriaux différents, sont corrélées à l'identité des maisons d'édition et au lectorat de la collection. 


\title{
Traduction et éditorialisation des récits sur la banlieue : paratexte iconique et transfert culturel
}

\author{
Béatrice Turpin \\ Université de Cergy-Pontoise
}

\section{Résumé}

Une première de couverture est un discours composite qui résulte de multiples choix éditoriaux. Elle doit refléter l'identité de la maison d'édition dont elle est la vitrine ainsi que celle de la collection qui ouvre des pistes de lecture. Elle doit également attirer le lecteur et lui donner une idée du contenu de l'ouvrage. Le présent article étudie l'éditorialisation de quelques récits sur la banlieue représentatifs de la littérature urbaine de la fin du $\mathrm{XX}^{\mathrm{e}}$ siècle et du début du $\mathrm{XXI}^{\mathrm{e}}$ siècle. L'analyse des publications françaises montre la prégnance d'un point de vue réaliste, avec une couverture qui peut être considérée comme une image arrêtée d'un scénario (souvent reprise d'un film qui a vu le succès du livre). Celle-ci tend par ailleurs à personnaliser l'ouvrage, avec la représentation en plan rapproché d'un narrateur/ personnage. Les éditions traduites tendent pour leur part à élargir le champ du regard en contextualisant le cadre historique ou spatial du récit ou, au contraire, effacent celui-ci. Le motif mis en scène, énigmatique, devient symbole ouvert sur de multiples interprétations. L'ouvrage, par là même, sort du cadre de la littérature dite «de banlieue». Ces deux tendances, emblématiques de choix éditoriaux différents, sont corrélées à l'identité des maisons d'édition et au lectorat de la collection.

Mots-clés : éditorialisation, première de couverture, motif, sémiotisation, réalisme, symbolisme

\section{Abstract}

A front cover is a composite discourse that results from multiple editorial choices. It must reflect the identity of the publishing house of which it is the showcase, as well as that of the collection which opens reading tracks. It must also attract readers and give them an idea of the content of the book. This article examines the editorialization of some suburban narratives representative of urban literature of the late $20^{\text {th }}$ and early $21^{\text {st }}$ centuries. The analysis of French publications shows the importance of a realistic point of 
view, with coverage that can be considered as a fixed image of a scenario (often taken from a film that has seen the success of the book). It also tends to personalize the book, with close-up representations of a narrator/ character. Translated editions tend on their part to widen the field of view by contextualizing the historical or spatial environment of the narrative or, on the contrary, erase it. The staged, enigmatic motif becomes a symbol open to multiple interpretations. The book, therefore, is outside the scope of "banlieue" literature. These two trends, emblematic of different editorial choices, are correlated with the identity of the publishing house and the readership of the collection.

Keywords: editorial treatment, front cover, key detail, semiotization, realism, symbolism

\section{Introduction}

Actuellement, et de plus en plus, les banlieues populaires en France sont associées à une culture jeune, hybride, comme peut l'être la langue parlée par la génération actuelle dans ces quartiers dont les grands ensembles constituent l'emblème (Turpin, 2012, 2015). Rap, graffs ou breakdance qui leur sont associés irradient les pratiques artistiques contemporaines ${ }^{1}$. Il existe un cinéma dit «de banlieue ${ }^{2}$ comme il y a une littérature dite «de banlieue» ${ }^{3}$, ce qualificatif renvoyant à des œuvres qui ont pour référent l'espace des cités d'habitation à loyer modéré (H.L.M.) situées à la périphérie des grandes villes. Ces récits ont été écrits/scénarisés le plus souvent « de l'intérieur» par des habitants de ces cités. Ils témoignent de la vie dans cet espace, mais aussi d'une manière de parler, la «langue des cités », qui mélange les idiomes et joue des fonctions cryptiques et ludiques des argots (Goudaillier, 1997, 2002, 2007, 2015). Certains auteurs ont maintenant acquis une audience internationale et sont traduits, avec la difficulté que représente ce langage particulier et un style parfois influencé par le rap (Vitali, 2012, p. 108-119).

Une traduction est à cet égard à la fois un texte, une écriture et un ouvrage. Comme texte à publier, elle s'accompagne d'un travail

1. Depuis 2006, le Paris Hip Hop Festival rend compte de cette dynamique (v. http://paris-hiphop.com/histoire/).

2. Représenté chaque année en France au festival CinéBanlieue, qui existe depuis 2006 (v. https://www.cinebanlieue.org/festival).

3. Littérature née dans cet espace, mais qui ne s'y réduit pas, au contraire : elle est révolte contre un espace ségrégué, parole ou cri, d'espoir ou de désespoir qui par là atteint à l'universel. Elle est aussi travail créatif sur la langue pour en repenser les associations convenues créées par l'habitude. On pourrait parler, plus exactement, de littérature postcoloniale en ce qui concerne le contexte français. 
éditorial dont elle est un élément. Elle est en effet souvent réalisée en fonction du contexte de réception et de l'éditeur qui accueille l'ouvrage (cf. Elefante, 2015). Ainsi, dans le paratexte, les voix se mêlent. La première de couverture est à la fois l'indice, l'icône et le symbole ${ }^{4}$ du texte lui-même, mais aussi de l'éditeur, de sa politique et de la collection dans laquelle il s'insère, liés à un ici et maintenant, à un lieu et à une histoire. Partie emblématique d'un ouvrage, elle est transposition d'un récit dans un système sémiotique visuel. Elle en représente les motifs ${ }^{5}$. Lors d'une traduction, comme d'ailleurs souvent lors d'une réédition, la scène est revue et les motifs réécrits. La saillance de motifs différents est un choix lié à un autre contexte éditorial, à la culture d'une maison d'édition, à celle de ses lecteurs destinataires de louvrage et aux attentes de ces derniers. Nous nous proposons ici plus particulièrement d'interroger ce travail de transposition dans l'édition des traductions de quelques récits sur la banlieue afin d'observer ce qui s'y inscrit et quels sont les enjeux qui peuvent guider un choix.

\section{Traduction et politique éditoriale}

La lecture ne se réduit pas à un transfert monologique et neutre entre un écrivain et son lecteur. Tous les «à-côtés» du texte créent un horizon d'attente qui contribue à sa signification. Ces «à-côtés» ou "paratextes", théorisés par Gérard Genette (2002 [1987]), vont de la lecture critique de l'ouvrage dans des journaux ou magazines à tout ce qui entoure le texte lui-même lors de sa publication : première et quatrième de couverture ou prière d'insérer, jaquette,

4. Nous utiliserons ces termes dans leur acception peircienne. L'icône introduit un rapport de similarité, "comme un dessin de triangle représente un triangle mathématique» (Peirce, 2002, p. 322), l'indice un rapport de fait et de contiguïté (ibid., p. 323), le symbole une association selon les préceptes de l'habitude (ibid., p. 310).

5. Dans l'analyse des premières de couverture, nous appelons motifs les référents du récit, accessoires et personnages. Un motif est un élément d'une scène, qui peut être représentée ou suggérée, par métonymie. La scène est elle-même partie du scénario (l'histoire racontée). Cette terminologie nous permet de marquer la distance qui peut exister entre le visuel et le textuel : la première de couverture n'est pas la reproduction du récit, mais son ouverture. Elle le représente, par métonymie ou par métaphore. Pour l'emploi du terme «motif», voir Turpin (2015, p. 128). Le «motif» peut être iconique, mais aussi textuel; il renvoie à un «pivot de référence». Entre le texte du roman et l'illustration de couverture, le motif esquisse un jeu de renvois qui a aussi une valeur performative : inciter à aller au-delà d'une opacité, de l'image-symbole arrêtée d'un récit. 
bandeau accompagnant l'ouvrage. Le paratexte est «ce par quoi un texte se fait livre et se propose comme tel à ses lecteurs, et plus généralement au public» (ibid., p. 7-8). Genette distingue à cet égard le péritexte, c'est-à-dire "les éléments éditoriaux qui entourent le texte", de l'épitexte, messages qui se situent à l'extérieur du livre : critiques, commentaires, interviews, etc. (ibid., p. 11). La première de couverture, tout comme la quatrième, relève de ce que Genette appelle plus précisément le péritexte éditorial. Il ajoute que la distinction entre épitexte et péritexte est parfois floue : une jaquette reproduisant l'affiche d'un film participe en effet, comme nous le verrons, de ces deux instances. Elle est à la fois de l'épitexte et du péritexte. Genette parle d'épitexte factuel (lié à un fait) - et c'est sans doute ce double statut qui fait sa force, lui donne valeur d'incitation à l'action (aller au-delà du seuil : lire, acheter pour lire).

Cette notion de paratexte permet de penser une sémiotique du discours éditorial qui insère l'écrit dans les éléments paratextuels qui concourent à sa signification. Le texte n'est en effet jamais clos sur lui-même dès lors qu'il entre dans un processus de communication, de lecture et d'interprétation qui transforme l'écrivant en écrivain, le texte en "récit» ou "roman» (dans le cas qui nous intéresse ici). Le paratexte est un élément d'une stratégie éditoriale qui donne sens à l'ouvrage, mais qui en fait aussi un produit culturel joignant transmission de sens et transaction. C'est par lui qu'un texte particulier devient œuvre, qu'un individu devient auteur, qu'un livre devient produit. L'énonciation éditoriale est d'emblée multiple, tissage de voix matérialisées dans un livre qui prend place dans un contexte historique et social déterminé qui guide à la fois l'émission et la réception. La première de couverture peut être à cet égard considérée comme l'emblème de ce processus que décrit très bien Clayton Childress dans son ouvrage Under the Cover:

For a story to become a novel-for it to be written by an author, make it through a literary agency, get into a publishing house and out the other hand, be promoted by publicity staff, be hand-sold in bookstores, be evaluated by reviewers and ultimately connected with by readers-it must be multiple. $(2017$, p. 3$)$

Dans la communication éditoriale se mêlent ainsi plusieurs voix, des lieux de production aux lieux de réception, avec leurs conditions. La politique éditoriale guide une stratégie d'interprétation. La première de couverture répond aux normes d'une maison d'édition 
et constitue son identité. Elle introduit le texte et est souvent le premier élément que voit le lecteur. Elle répond également à des normes propres à un genre. Ainsi reconnaît-on un roman policier aux caractéristiques de sa couverture qui se décline le long d'une collection, dans un système de ressemblances et d'oppositions avec les autres collections d'un éditeur ou d'autres éditeurs : importance de la photographie (opposée à l'illustration), du noir et blanc déclinant le jeu de l'ombre et de la lumière avec prégnance de deux autres couleurs, le rouge, indice du sang, et le jaune, de la lumière. Ce code à la symbolique forte, omniprésent dans les romans policiers occidentaux, pourra cependant être revu pour marquer des différences (d'éditeurs ou d'espaces géographiques). Ainsi, chez Philippe Picquier, éditeur spécialisé dans les livres traduits venant d'Extrême-Orient, ces codes ne sont-ils plus obligatoirement de mise pour faire ressortir, par le choix de motifs graphiques, le thème de l'Orient en même temps que l'identité de la maison (cf. Éditions Philippe Piquier, s.d.). La première de couverture traduit ici la stratégie éditoriale de l'éditeur, sa stratégie de reconnaissance, la manière dont il se place par rapport aux autres éditeurs et par rapport à son lecteur. Elle donne également du sens à l'ouvrage, avant même que celui-ci ne soit lu.

Un autre genre fortement marqué est celui des livres pour enfants ou pour adolescents. Ici, la photo fait place à l'illustration, l'illusion réaliste s'ouvre sur le rêve. Les codes esthétiques sont ceux de l'imagination, avec une liberté et une fantaisie du trait, des couleurs vives et variées. Ces codes signent l'identité d'un genre et d'un lectorat par delà les traductions. Les stratégies de communication et de reconnaissance sont basées avant tout sur la séduction visuelle. Pour un roman destiné à un public d'adolescents, le dessin demeure souvent, mais il est traité avec de larges aplats qui créent une illusion de photographie.

Dans le présent article, nous étudions comment ces processus de sémiotisation opèrent pour les premières de couverture de romans sur la banlieue et dans certaines de leurs éditions en langue étrangère. Notre corpus est constitué principalement d'ouvrages d'auteurs traduits dont l'essentiel de l'ouvre est constitué de romans ayant pour cadre les quartiers H.L.M. situés à la périphérie des grandes villes et pour sujet le récit de parcours de vie. Nous distinguerons deux périodes. La première se situe dans les années 1980. C'est celle de la Marche contre le racisme et pour l'égalité (rebaptisée du 
stigmatisant «Marche des beurs» par certains journalistes) $)^{6}$, celle des récits de vie d'enfants d'immigrés et de la tension ressentie entre deux cultures. Les écrits qui la caractérisent sont pour la plupart des témoignages autobiographiques, avec un référent politique lié à la fin de la colonisation. Citons Azouz Begag, qui raconte son enfance dans les bidonvilles de la banlieue lyonnaise dans Le Gone du Chaâba (1986)7, et Mehdi Charef, qui publie en 1983 Le Thé au harem d'Archi Ahmed, "[u]ne cité H.L.M. Sur les murs : graffitis, slogans, appels de détresse, dessins obscènes. Madjid vit là. Il est fils d'immigrés, paumé entre deux cultures, deux langues, deux couleurs de peau, et s'invente ses propres racines, ses attaches" (Charef, 1988, quatrième de couverture). La deuxième période se situe au tournant de ce siècle; elle est marquée par un accroissement des tensions dans les quartiers populaires, notamment après les émeutes de 2005 et l'état d'urgence qui a suivi ${ }^{8}$. La banlieue devient un objet d'attention médiatique dont vont profiter certains jeunes pour faire entendre leurs voix à travers l'écriture, sous ses multiples formes, du cinéma à la littérature, avec des œuvres engagées socialement. À travers ces œuvres s'affirme une culture propre aux espaces urbains (influence du hip-hop et du rap notamment). Citons les écrits de Rachid Djaïdani et de Fä̈za Guène, qui furent d'emblée des best-sellers traduits à l'étranger. Le collectif Qui fait la France?", pour lequel la littérature est un engagement sociétal, est également représentatif de cette seconde période :

la littérature s'envisage comme un verdict sur la société et les mentalités pour lesquelles elle est écrite. [...] [Les auteurs du collectif] ont une empathie pour les banlieues, les enfants d'immigrés,

6. Appellation faite à partir d'une verlanisation de «reub»: «arabe», cela alors même qu'à travers cette marche toute une génération née en France réclamait le droit à une égalité de traitement.

7. Gone est l'équivalent de "gosse» en patois lyonnais; Chaâba désigne un quartier de Villeurbanne, en banlieue lyonnaise, sur lequel fut implanté un bidonville ainsi dénommé par les habitants - de l'arabe dialectal : «lit desséché d'un oued» (Wiktictionnaire, n.d., n.p.).

8. À la suite de la mort par électrocution de deux adolescents poursuivis par la police alors qu'ils revenaient d'un entraînement sportif éclatent en banlieue parisienne des émeutes qui s'étendront bientôt à la France entière, amplifiées par les déclarations de dirigeants politiques et l'incendie d'une mosquée à la suite d'un tir de grenade par la police (v. Mauger, 2006).

9. Formé en avril 2007, il regroupe Karim Amellal, Jean Eric Boulin, Khalid El Bahji, Dembo Goumane, Faïza Guène, Habiba Mahany, Samir Ouazène, Mabrouck Rachedi, Mohamed Razane et Thomte Ryam (Fumigène, 2007, n.p.). 
les invisibles, les précaires, les méprisés, les indignés, les souffrants, qui donnent cette couleur à leurs écrits. (Mohamed Razane, dans Fumigène, 2007, n.p.)

Mentionnons également Rachid Santaki, auteur d'un récit en partie autobiographique, La petite cité dans la prairie (2008), qui s'attachera, après avoir signé ce premier ouvrage, à mettre en scène la face «noire ${ }^{10}$ de trajectoires de vie dans les banlieues populaires ${ }^{11}$.

C'est à partir de sa couverture qu'un ouvrage se donne à voir. Celle-ci est à la fois présentation, incitation à la lecture et support de significations soumises par le graphiste et l'éditeur. Les variations des premières de couverture selon les réimpressions en actualisent les gammes. Elle est également à la fois traduction et interprétation du texte. Elle le traduit dans une autre forme sémiotique, en module le sens à partir de multiples possibles par des choix graphiques et éditoriaux réalisés par rapport à un contexte de lecture. Dans le passage d'une langue à une autre, ce contexte renvoie à une histoire politique et sociale différente et à un autre lectorat.

Nous partirons des exemples suivants, représentatifs des mutations observées dans la traduction et l'éditorialisation de récits sur la banlieue :

- Le Gone du Chaâba d'Azouz Begag $(1986,1998,2005)$ et sa traduction anglaise par Naïma Wolf et Alec G. Hargreaves, parue en 2007;

- Le Thé au harem d'Archi Ahmed de Mehdi Charef (1983, 1985,1988 a et $1988 b^{12}$ ) et sa traduction anglaise de 1989 par Ed Emery;

- Kiffe kiffe demain de Faïza Guène $(2004,2005,2010)$ et sa traduction anglaise de 2006 par Sarah Adams;

10. Nous employons ce terme en référence au «roman noir» qui pourrait correspondre à bon nombre de récits de banlieue : «le roman "noir” [...] enracine les crimes dans les circonstances sociales dans lesquelles ils sont commis. Ce n'est plus l'individu seul qui est criminel, c'est le monde de souffrance, de misère, de violence et de corruption dans lequel nous vivons qui produit les individus criminels, ce monde que la loi et la justice recouvrent, sans l'organiser» (Manotti, 2007, p. 107).

11. De Les anges s'babillent en caillera publié aux éditions Moisson rouge en 2011 au titre Les princes du bitume publié aux éditions Jigal en 2017, on dénombre une dizaine de titres de Santaki qui se situent entre roman noir et roman policier. Cet auteur n'est à notre connaissance pas traduit.

12. Lédition de 1988 fut suivie de tirages ultérieurs, avec un changement de la première de couverture (notée alors 1988b). 
- Mon Nerf de Rachid Djaïdani $(2004,2005)$ et sa traduction italienne de 2011 par Ilaria Vitali;

- Viscéral de Rachid Djaïdani $(2007,2008)$ et sa traduction italienne de 2009 par Ilaria Vitali.

Entre le récit source et sa traduction, nous pouvons observer deux types d'opérations: 1) une contextualisation généralisante, avec élargissement et précision sur le contexte de l'ouvrage; 2) une symbolisation, avec passage du référentiel à l'onirique ou au symbolique. Il n'y a pas, dans ce dernier cas, ajout d'une précision : le sens s'ouvre à l'indéterminé.

\section{Une contextualisation généralisante ${ }^{13}$}

La traduction des œuvres du corpus envisagé tend à contextualiser dès la première de couverture le cadre du récit, historiquement dans Le Gone du Chaâba d'Azouz Begag, spatialement dans Kiffe kiffe demain de Faïza Guène.

Le Gone du Chaâba est d'abord édité au Seuil en 1986 dans la collection Point-Virgule, collection de poche un peu «décalée» par rapport à la collection Points plus classique, avec notamment des livres destinés à un jeune lectorat. La première de couverture de ce récit en grande partie autobiographique reprend le motif central de l'ouvrage : l'enfant, narrateur et personnage. Le choix esthétique de cette première couverture est celui d'un dessin de type réaliste qui évoque les recueils de l'époque destinés à un jeune public ${ }^{14}$. Le personnage mis en scène est le double en miroir du lecteur supposé, "un écolier», représenté de manière figurative : les indices d'appartenance sociale sont gommés. Le thème de l'école, évoqué par un pupitre et un carnet à spirales de couleur jaune, ouvre sur une séquence du récit : celle de la remise des notes, quand le narrateur apprend qu'il est deuxième de sa classe ${ }^{15}$. Seule la quatrième de couverture fournit de l'information sur le contexte de l'histoire.

13. Nous remercions les éditeurs suivants des autorisations de reproduction qu'ils nous ont accordées pour les images des couvertures reproduites dans les pages qui suivent : Les Éditions Hachette, Fayard, Paris; Les Éditions du Seuil, Paris; Giulio Perrone, Rome; Houghton Mifflin Harcourt Publishing Company, New York; Profile Books, Londres; University of Nebraska Press, USA.

14. La couverture en question peut être consultée à l'adresse suivante : http:// images-chapitre.com/ima2/original/725/339725_9418151.jpg.

15. "Je saisis mon carnet à pleines mains, avec une émotion si intense que j'ai envie de pousser un cri» (Begag, 2005, p. 86). 
La sortie du film en 1997 et son succès amèneront des rééditions avec en première de couverture une photo extraite du film. Le récit reparaît d'abord en 1998 au Seuil dans la collection Cadre Rouge avec une jaquette illustrée, puis en 2005 dans la collection Points : roman ${ }^{16}$. Ces rééditions s'adressent à un lectorat élargi en raison du succès $\mathrm{du}$ film. La photo ${ }^{17}$ représente le narrateur et personnage principal du film (l'acteur Bouzid Negnoug dans le rôle d'Omar). Le contexte social est ici suggéré : les motifs de la pauvreté et les conditions de vie rudimentaires sont inscrits sur les vêtements que porte l'enfant (taches sur un manteau en drap rustique, tee-shirt bâillant, chaussettes sans élastique). Cette pauvreté se lit également à partir de l'environnement dans lequel il se trouve : il lit dans la rue, assis sur des marches de bois blanchi. Le fond est gris, de même que les vêtements. L'enfant est concentré sur son livre, concentration qui renvoie à sa stature de bon élève (et à sa future réussite). Par la composition en diagonale descendante qui suit le regard du personnage en train de lire et par une prise de vue en plan semi-rapproché, le lecteur est mis en situation de spectateur et de témoin. La présence d'une photo qui marque un moment d'une histoire ouvre sur la diégèse et, par ses motifs, sur le contexte social. La quatrième de couverture est quant à elle actualisée avec

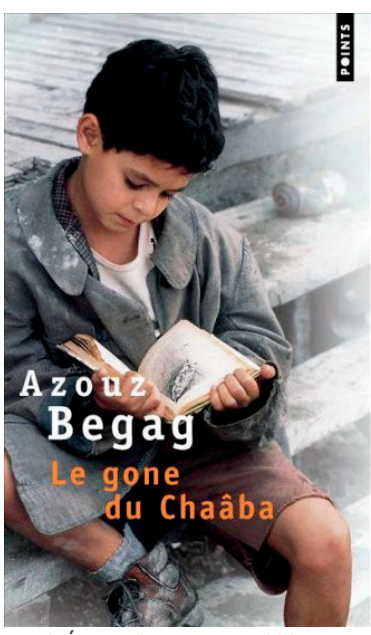

๑) Éd. du Seuil, Points, 2005 mention de la nouvelle fonction de ministre délégué à l'égalité des chances d'Azouz Begag. La sortie du film donne ainsi un nouvel élan à l'ouvrage et pousse l'éditeur à sortir du récit-témoignage destiné à un jeune public pour promouvoir le roman à partir de son épitexte cinématographique convoqué pour signifier le succès du livre et donc implicitement son intérêt. L'épitexte en devenant péritexte confère à ce dernier une valeur argumentative qui renforce sa valeur marketing. Le plan rapproché centre là encore le propos sur l'enfant photographié : le récit est ainsi toujours présenté comme récit de vie d'un individu, l'auteur-personnage.

16. L'ouvrage sera réédité en 2001 dans la collection Points Virgule avec la même photo.

17. Les noms des photographes, lorsque connus, sont indiqués en fin d'article, dans la référence bibliographique des romans en question. 
Le contexte social, seulement suggéré dans ces dernières éditions françaises, sera par contre mis en avant dans la traduction en anglais américain de Naïma Wolf et Alec G. Hargreaves, parue en 2007 sous le titre Shanty-town Kid [litt. : L'enfant des bidonvilles] avec comme sous-titre, en petits caractères et entre parenthèses, le titre français. L'éditeur a voulu effacer le caractère énigmatique de l'appellation et nommer sans ambiguïté l'espace dans lequel se déroule le roman. La couverture reproduit une photographie documentaire d'un bidonville emprunté à la banque d'images Corbis, symbole du quartier du Chaâba lui-même, situé au bord du Rhône, avec son terrain boueux. Il s'agit d'une édition universitaire publiée

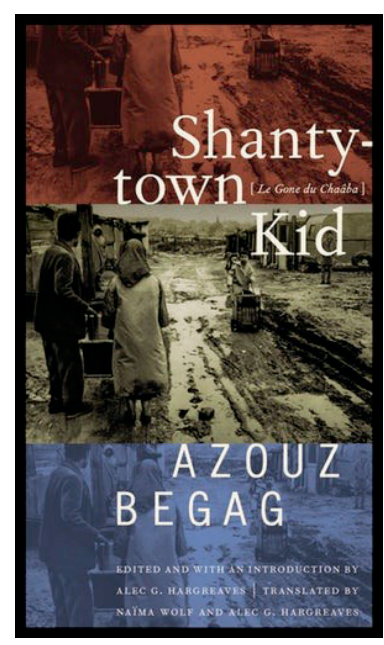

(c) Univ. of Nebraska Press, 2007 dans le cadre d'études francophones, qui cherche à illustrer par le texte et l'image la situation des travailleurs immigrés en provenance d'Afrique du Nord et de leur famille dans les campements de fortune situés dans les périphéries urbaines. La transposition du visuel montre la différence du regard, déporté d'un destin individuel vers un destin collectif : celui des travailleurs algériens et de leurs conditions de logement dans l'après-guerre, anticipant la construction des grands ensembles (le récit de Begag marque le passage d'un espace à l'autre, avec le déménagement de la famille du bidonville aux immeubles modernes).

Dans l'édition américaine, la première de couverture marque le contexte politique et culturel du récit. En contextualisant sur l'histoire sociale d'un pays étranger, elle illustre le texte, mais le commente également en répétant, comme en écho, de l'image au récit ou du récit à l'image, cette activité de commentaire que constitue la traduction d'un texte d'une langue à une autre, commentaire élaboré en fonction d'un destinataire. Dans l'édition française, l'accent est mis implicitement sur la lecture et l'importance de l'école dans l'ascension sociale alors que l'édition américaine met en scène les bidonvilles en contexte postcolonial. Les premières de couverture sont donc de ce point de vue, comme souvent les quatrièmes, un commentaire de l'œuvre qui dessine un point de vue particulier en fonction d'un destinataire et du but de la traduction. 
Ce passage de l'individuel au collectif ou du particulier au général dans la traduction correspond à une différenciation du lectorat auquel s'adressent le roman français et sa version américaine. Cette différenciation se retrouve entre les deux éditions, en langue française et en anglais, du récit de Fä̈za Guène Kiffe kiffe demain, avec passage d'une illustration centrée sur la psychologie du personnage à une illustration qui situe l'ouvrage dans son contexte social et spatial. La première édition, parue chez Hachette Littérature en 2004 avec une jaquette illustrée représente une jeune fille rêvant derrière une fenêtre. La réédition dans la collection Le livre de Poche en 2005 comporte quant à elle en plan très rapproché, une photographie de l'écrivaine, souriante et séductrice, qui fait face à son lecteur qui se trouve impliqué par ce regard. Par ce resserrement du champ du regard, l'édition de 2005, en se faisant témoignage, voire conversation, cherche à élargir son lectorat. Nous ne sommes plus témoins des pensées du personnage, nous sommes avec lui.

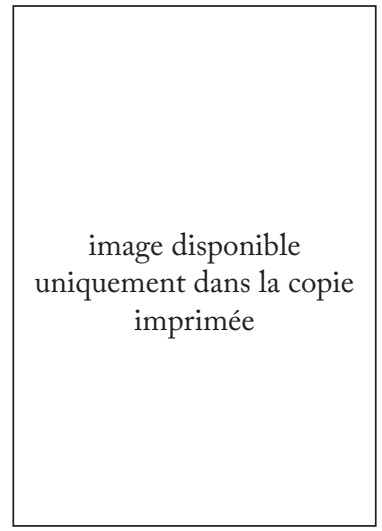

(c) Hachette Littérature, 2004

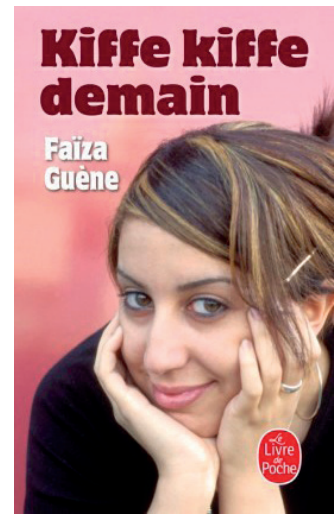

(c) LGF, Le Livre de Poche, 2005

Cette personnalisation de la couverture est abandonnée dans l'édition traduite en anglais de 2006. La couverture (voir page suivante) présente un personnage en plan de demi-ensemble au visage caché par sa chevelure. Deux immeubles l'encadrent, les lignes de fuite mettant en valeur le titre dont la partie d'argot français dérivée de l'arabe n'est pas traduite, mais expliquée en quatrième de couverture. Les commentaires, sur les deux faces, affichent l'ouvrage comme un récit de l'adolescence, ce qui lui donne une portée documentaire, crédibilisée par la biographie de l'auteure placée en 
quatrième de couverture, qui la présente, à côté de sa photo, comme étudiante en sociologie dans une université parisienne. On retrouve ici cette contextualisation plus générale mettant en valeur l'intérêt social et documentaire du roman plutôt qu'une personnalisation de type autobiographique.

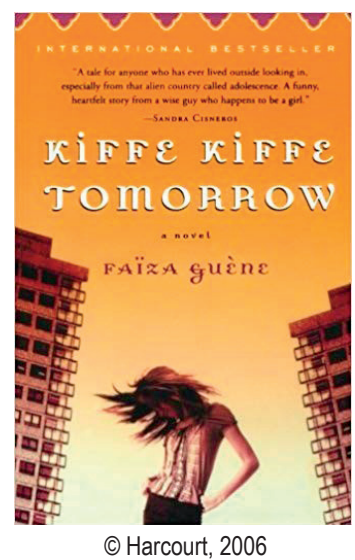

\section{Une ouverture surl'indéterminé}

Nous pouvons distinguer un autre processus sous-jacent à la conception du péritexte iconique. Il s'agit d'un changement de dimension : l'image de couverture de l'édition traduite ne contextualise plus la dimension documentaire du récit, mais met l'accent sur un motif qui, par ses connotations, en condense l'atmosphère. Il s'agit donc dans ce cas d'une transposition, avec passage du référentiel à l'onirique ou au symbolique. La généralisation ne porte plus sur le contexte, mais aboutit à créer de l'indéterminé. Plusieurs paratextes iconiques illustrent cette seconde tendance, dont ceux du roman de Mehdi Charef, Le Thé au harem d'Archi Ahmed, et des romans Mon Nerf et Viscéral de Rachid Djaïdani.

Le récit de Mehdi Charef aborde la question du déracinement et du tiraillement entre deux cultures. L'espace du récit est celui d'une cité H.L.M. sur fond de trafic de drogue et de violence, avec une vision désespérée devant un avenir sans promesse. Le titre fait référence à l'école et aux difficultés de compréhension linguistique et donc d'apprentissage. Le mélange des langues, la métacoupure et la réinterprétation d'une langue par l'autre sont métonymies du récit et symbolisent cet écartèlement entre deux mondes que met 
en scène la diégèse ${ }^{18}$. Louvrage parut en 1983 au Mercure de France dans la prestigieuse collection Bleue. Selon les codes de sobriété et de prestige propres aux grandes maisons d'édition en France, le nom de la collection renvoie à la couleur de la couverture, signant une identité visuelle forte, réduite à son plus simple dénominateur : la couleur (comme la Blanche de Gallimard). La couverture ne dit rien de plus que le prestige et l'évidence de la qualité ${ }^{19}$. Le livre se présente d'emblée comme «littérature ». La couverture est cependant doublée d'une jaquette avec une photographie reproduisant l'affiche du film après la sortie de celui-ci en 1985, sous un titre légèrement différent : «Le Thé au harem d'Archimède», phoniquement plus simple et donc plus commercial. Lillustration de la jaquette met en exergue trois motifs : "une bande de jeunes», "une cité H.L.M.» et, au premier plan, une botte de type western et une basket liserée de bleu-blanc-rouge, indices métonymiques des deux principaux personnages du récit, Majid et Pat, et de leur amitié ${ }^{20}$. Nous sommes ici dans le référentiel, avec des motifs qui reprennent de manière figurative des éléments du récit pour les mettre en scène dans un scénario d'actions: il s'agit d'un instantané, d'une coupure dans un récit dont la lecture promet la continuité. Les choix référentiels et esthétiques font penser à un livre d'aventure pour adolescents. Comme pour l'ouvrage d'Azouz Begag, la jaquette est destinée à doper les ventes, à élargir le lectorat. Sa fonction est avant tout publicitaire. Elle devient affiche, destinée à être visible, reconnue (v. Reuter, 1981, p. 107 : «La jaquette des livres "nobles", qui est récente, se fonde sur des études montrant que $40 \%$ de la vente des livres se fait en vitrine»).

Le livre fut réédité quelques années plus tard dans la collection Folio de Gallimard, avec deux éditions successives au visuel différent. Dans la première édition de poche, parue en 1988, le visuel représente une image composite rappelant les éléments de la jaquette de la collection Bleue. Il met en avant le mélange des cultures avec trois motifs prégnants : un chameau, des barres d'immeubles, une

18. Le titre renvoie à un souvenir d'enfance de l'auteur, à une erreur d'analyse de la chaîne acoustique "théorème d'Archimède» réinterprétée par «thé au harem d'Archi Ahmed» par un enfant maghrébin, camarade de classe de Mehdi Charef. 19. On peut consulter la couverture à http://www.gallimard.fr/Catalogue/ MERCURE-DE-FRANCE/Bleue/Le-the-au-harem-d-Archi-Ahmed.

20. On peut consulter la couverture à l'adresse suivante: https://www. livrenpoche.com/le-the-au-harem-d-archi-ahmed-e340143.html. 
botte évocatrice de la mode «jeune » rappelant celle qui est présente sur la jaquette et sur l'affiche du film. Les sèmes actualisés dans ce montage renvoient à deux espaces distincts, évocateurs des deux cultures entre lesquelles sont écartelés les personnages, mais aussi à des choix esthétiques ancrés dans l'époque et assez datés actuellement (montage d'éléments disparates, présence d'une botte santiag). Les rééditions abandonnent ces choix et situent la représentation dans la culture jeune des années 1990, pour mettre en scène deux grapheurs en acte et vus de dos en train de taguer un mur bétonné - métonymie d'un motif du récit, mais aussi autre acte d'écriture dont le lecteur est ainsi comme témoin, invité à passer audelà du mur : à la lecture.

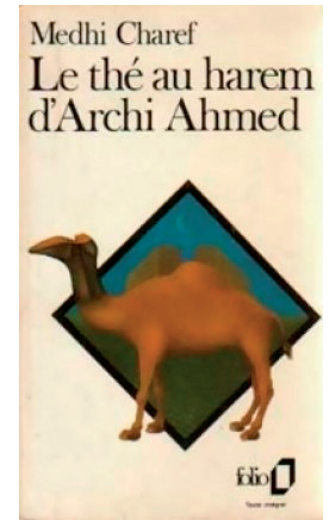

๑ Éd. du Seuil, Folio, 1988a

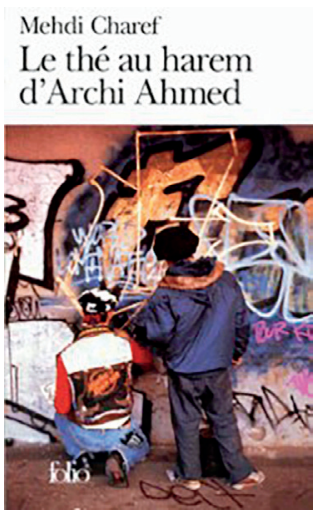

๑ Éd. du Seuil, Folio, 1988b

L'édition anglaise publiée en 1989 chez Profile Books adopte un visuel composite et très coloré qui reprend le thème du graff pour en faire l'unique illustration. Ouvert sur la littérature-monde, l'éditeur publie des ouvrages emblématiques de la culture contemporaine, ce dont atteste cette première de couverture qui comporte par ailleurs une citation de l'écrivain britannique Hanif Kureishi, auteur du scénario du film My Beautiful Laundrette de Stephen Frears et du récit The Buddha of Suburbia traduit dans une vingtaine de langues, qui eux-mêmes traitent de thèmes communs avec la littérature urbaine : difficulté pour une jeunesse de vivre une double culture, sur fond de tensions raciales et de culture pop des années 1970. La couverture reproduit l'univers du graff, avec deux inscriptions qui renvoient au personnage du narrateur, «Moi» et «Majid». 
Lillustration, composite comme peuvent l'être les graffs, en pleine page, devient ainsi symbole du récit dans son ensemble. Le graff n'est plus considéré dans sa dimension de tag, mais comme forme artistique qui rappelle la dimension onirique de certains passages du récit. Nous pouvons également relever la présence de deux motifs stéréotypés de la France et plus spécifiquement de Paris : la Tour Eiffel et le Sacré-Cœur, deux emblèmes souvent présents sur les couvertures de guides touristiques ou les affiches d'agence de voyages: ne faut-il pas y voir, en même temps qu'un ancrage spatial, une invitation

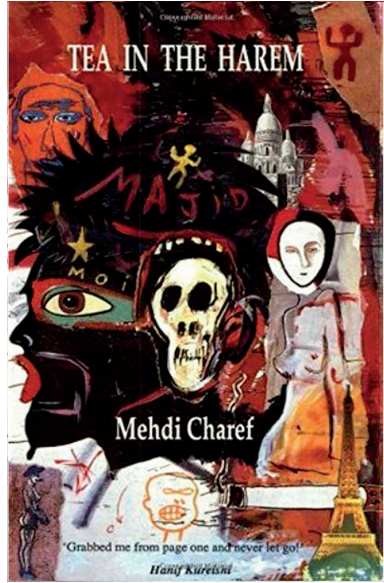

C Profile Books, Londres, 1989 à voyager le temps d'une lecture et l'inscription d'un label qui rappelle la fonction commerciale des premières de couverture illustrées. Le texte de la quatrième de couverture, quant à lui, semble décliner les motifs illustrés sur la première : Paris, France, suburb/s, surroundings, Moroccans, Algerians, Arabic, French, Arabs, Beur, culture/s, secondgeneration, portrays, mother's, fiction, reality, language, rejects, insults, invective, growing, housing, poetic, beauty, Madjid, school, slang.

La première de couverture de l'édition anglaise peut être considérée comme une réinterprétation du récit dans une fresque colorée qui en transpose stylistiquement les motifs. Elle traduit l'aspect pionnier de Profile Books qui fait du graff une véritable œuvre d'art et transpose des motifs narratifs qui vont du réalisme à l'onirisme comme le fait le récit. Alors que l'onirisme se projette dans les codes culturels du hip-hop, les indices référentiels renvoient ici aux codes de reconnaissance d'un pays et donc à la langue d'origine de l'ouvrage traduit. Cette figuration évoque le travail de dislocation et de recombinaison phoniques présent dans le titre français, mais gommé dans la traduction anglaise, sans doute afin de garder le même titre que le film et de profiter ainsi de son effet publicitaire. Profile Books, comme Serpent's Tail qui l'a maintenant rejoint, a pu bousculer l'édition traditionnelle anglaise par le choix des ouvrages publiés, mais également par des choix esthétiques affirmés, avec des couvertures toujours très graphiques. Dans le cas de Tea in the Harem, la couverture n'est plus l'image arrêtée d'un scénario, 
elle ne renvoie plus à un moment du texte, mais à son ensemble, à son esprit tout autant qu'à l'identité de l'éditeur. Nous avons appelée «transposition onirique» cette transposition sans scénario qui, destinée ici à un lecteur anglophone, annonce un espace géographique autre: l'espace parisien, avec ces indices d'un autre lieu, d'une autre langue que sont les deux monuments représentés.

Ce travail de transposition est également présent dans les traductions des romans de Rachid Djaïdani Mon Nerf (2004, 2005) et Viscéral (2007, 2008). Dans les versions françaises, la première de couverture est très personnalisée, avec chaque fois la photo de l'auteur, reconnaissable, quoique floue sur la première édition de Mon Nerf. Celui-ci y est représenté vêtu du survêtement type du jeune des cités, capuche sur la tête, à la fois protection et manière de se cacher. Il fait face au lecteur, le regard dirigé vers lui. Le flou cependant dépersonnalise et a une valeur généralisante : l'homme représenté est un type plus qu'un individu. Cette première de couverture renvoie davantage à un stéréotype qu'au personnage précis décrit dans Mon Nerf, avec ses sandalettes, son bermuda et son débardeur (cf. Djaïdani, 2004, p. 55). Elle affiche une continuité entre le premier récit de Djaïdani, Boumkoeur (1999), dont la couverture représentait aussi l'auteur, et cherche à promouvoir l'ouvrage par ce rappel, également présent sur un bandeau orangé, avec l'inscription "par l'auteur de Boumkoeur». Les motifs mis en avant sont ceux du «jeune de banlieue» et celui de la "cité» ${ }^{21}$ - alors que l'ouvrage va bien au-delà de ces thématiques.

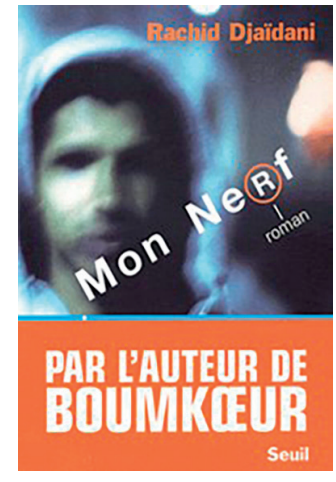

๑) Éd. du Seuil, Cadre Rouge, 2004

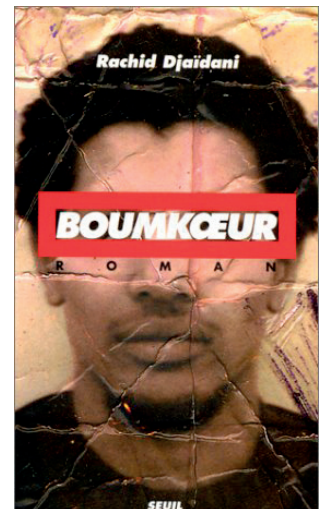

(c) Éd. du Seuil, Cadre Rouge, 1999

21. Ce qu'atteste le commentaire de l'éditeur sur la quatrième : «Rachid Djaïdani lève un voile sur l'envers invisible des cités». 
Seuls quelques éléments graphiques déportent ce sens, avec la reprise d'éléments présents dans le corps du récit, notamment autour du ${ }^{\circledR}$ de NeRf/Roman/RER et la présence d'une ligne bleue qui traverse la page et se termine sur la quatrième de couverture qui évoque quant à elle ce qui peut former l'unité narrative de ces deux premiers récits : le battement d'un cour avec ici l'évocation d'un état psychique, mélange d'ennui, d'angoisse et de rage.

L'édition de poche de Mon Nerf, parue l'année suivante, en 2005, joue quant à elle sur l'incongruité pour retenir l'attention, avec toujours la représentation de l'auteur, ici avec une coiffe d'Amérindien. Celle-ci renvoie au mal-être du personnage, Mounir: "Moon Air, souffle lunaire»: «un gars passionné par les Indiens et les trains, un gars circoncis de travers et qui sur terre ne put s'enivrer à pêcher les arômes de la chair» (Djaïdani, 2004, p. 158). On peut cependant trouver la photo plate par rapport à l'inventivité de la prose de l'auteur, @éd. du Seuil, Points, 2005 juste intrigante et par là incitant à lire le récit.

La photo de Rachid Djaïdani se retrouve sur la couverture des deux éditions de Viscéral, paru d'abord en 2007 dans la collection Littérature, puis en 2008 dans la collection Points. La photo est identique dans les deux éditions, mais avec des éclairages plus contrastés dans l'édition de poche (voir page suivante). Le visage de Djaïdani est en partie dans l'ombre, et la photo est prise en légère contre-plongée, sur un fond bleu-gris : ce portrait d'homme apparaît ainsi, avec une part de mystère, magnifié tel un buste d'Apollon. Le regard implique le lecteur qui s'attend à un récit dont ce personnage photographié serait le centre. Plusieurs passages du roman, dressant le portrait du boxeur Lies, semblent en être le commentaire suggérant, dans ce récit à la troisième personne, une identité entre Rachid Djaïdani, lui-même boxeur, et son personnage :

le visage émacié, taillé dans le reflet d'un prince du désert. Des pigments de cannelle se mêlent à sa peau mate. Son regard persan vise droit dans les pupilles, il se dégage de lui une énergie compacte (2010, p. 14-15)

le visage dévoré par une ombre douce, son regard est clinquant. (2010, p. 138). 
Le titre du roman, qui surplombe le portrait photographié, semble en signifier à la fois la souffrance et la puissance.

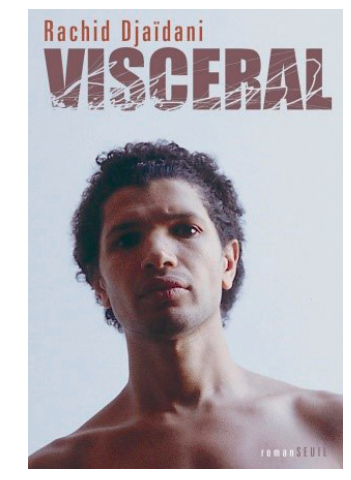

๑) Éd. du Seuil, Cadre Rouge, 2007

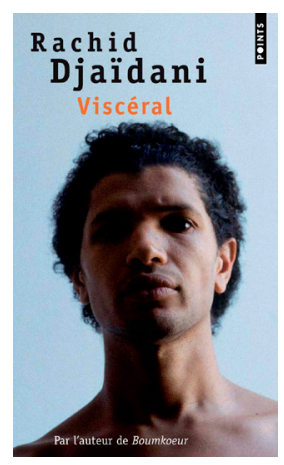

๑ Éd. du Seuil, Points, 2008

Dans ces différentes éditions, la première de couverture dessine un horizon d'attente en ce qui concerne le contenu du roman. Cet horizon d'attente est subordonné à un objectif marketing : après 2005 le thème de la banlieue et celui du «jeune de banlieue» sont abondamment traités dans les médias et intéressent les lecteurs de journaux ou magazines en même temps qu'émergent les voix de la banlieue à travers romans et œuvres cinématographiques (v. Carpenter et Horvath, 2015). L'éditeur choisit d'inscrire les récits de Rachid Djaïdani dans cette dynamique qui oriente la lecture, mais à laquelle le texte échappe finalement : la couverture n'est ici qu'une projection du récit, qui ne peut se montrer après-coup que déceptive par rapport à un texte qui fut pourtant sa promesse preuve s'il en est que la couverture ne ferme pas le sens.

Les éditions italiennes parues chez Giulio Perrone abandonnent cette personnalisation. Les photographies acquièrent une part de mystère. Sur la couverture de Ritratto di un ragazzo da buttare alle ortiche (Djaïdani, 2011), la traduction de Mon Nerf, le bas de deux jambes, coupé aux mollets, apparaît au centre de la page, sur fond bleu pervenche. Ces jambes sont terminées par deux pieds joints chaussés de souliers de ville. Ceux-ci semblent pendre audessus du vide. L'image intrigue. On y perçoit une certaine féminité. Elle évoque le retour sur soi, la réflexion, la solitude et le silence. Et c'est bien de cela qu'il s'agit dans ce récit à la première personne : du cheminement d'une pensée lors d'un déplacement en 
RER jusqu'au cabinet d'un psychanalyste, avec arrêts sur souvenirs ou traumas - avec en toile de fond, non loin d'une cité H.L.M., un quartier pavillonnaire avec son racisme et sa peur du différent, «fragiles maisonnettes qui nous emprisonnent au milieu de nulle part» (Djaïdani, 2004, p. 18). La première de couverture traduit ici par l'image l'état d'esprit du narrateur: «Le temps ne me collera sans doute jamais une ride, car j'ai le sentiment d'être un éphémère touchant du bout du doigt sa destinée du six pieds sous terre» (ibid., p. 77); «mon arôme est celui de l'encre noire. Mes ailes m’ont été livrées sans le mode d'emploi. Je suis un silence qui me griffe l'épiderme. J'ai la couleur de l'hiver sans la légèreté du flocon » (ibid., p. 78). On retrouve cette réinterprétation dans le rapport entre les deux langues figuré par le titre italien ${ }^{22}$ : Ritratto di un ragazzo da buttare alle ortiche [litt. : «Portrait d'un garçon à lancer aux orties»] qui renvoie lui-même à la narration comme «autoportrait d'un jeune gens tout juste bon pour les mauvaises herbes» (ibid., p. 77). L'idée du "Nerf», de la peur de la castration ou de la circoncision vécue comme castration, est transposée dans ces symboles que sont ces pieds suspendus au-dessus du vide, qui eux-mêmes renvoient au récit suivant de Rachid Djaïdani, Viscéral, déjà paru l'année de la traduction : au moment du récit où le boxeur chancelle, «les pieds dans le vide tel un pierrot la lune» (2008, p. 126).

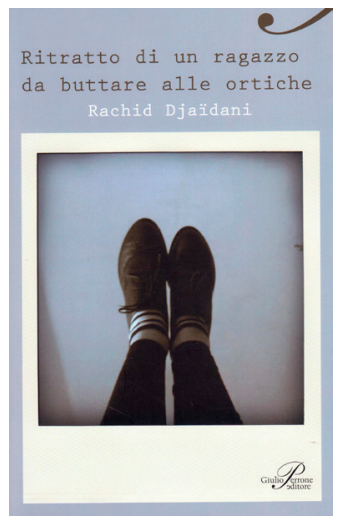

(c) Giulio Perrone, 2011

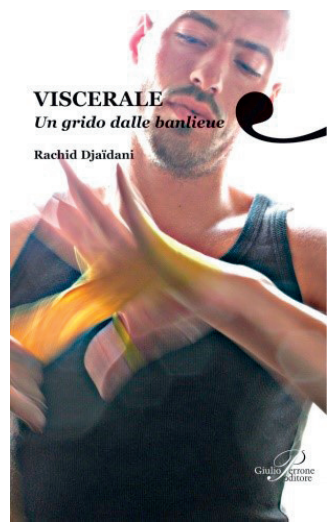

(c) Giulio Perrone, 2009

22. Au sujet de cette traduction, voir Vitali (2013). 
Dans Viscerale, la traduction italienne (Djaïdani, 2009), la photo de l'écrivain disparaît (voir page précédente). Comme dans Ritratto di un ragazzo da buttare alle ortiche, la première de couverture a un caractère énigmatique. Il s'agit d'un moment arrêté qui dessine une calligraphie dont le sens ne se dit pas d'emblée. Au centre de l'image, une main en mouvement semble tournoyer. Le visage du personnage, en contre-plongée, paraît concentré sur ces mains, sans que ne s'y lise une autre expression. L'arrêt de l'image ne laisse rien présager de la suite, sauf peut-être un mouvement, une répétition. Le titre lui-même ne permet pas d'en préciser le sens et ne peut donner lieu qu'à des hypothèses interprétatives, dont le sous-titre «Un grido dalle banlieue» [un cri de la banlieue] vient juste préciser le cadre, la banlieue dans cette traduction parue en 2009, quelques années après que les banlieues françaises aient été sous les feux de l'actualité. Seule la lecture du texte pourra induire des réponses aux questions posées par le titre et par la photographie, sans en épuiser le sens. Le titre? Le nom d'un manuscrit dont on ne connaît pas la fin, mais probable mise en abîme du texte de l'auteur. L'illustration? Un renvoi à ce manuscrit et à ce passage qui fait écho avec la première de couverture : "Lies se ventile sa belle gueule avec le manuscrit»? Non : une main qui tournoie pour enrouler les bandes protectrices autour du poignet d'un boxeur, bandes oubliées avec lesquelles se pendra un prisonnier dans le récit. Quant au visage, il ne répond pas aux stéréotypies permettant d'induire une origine géographique. Il pourrait être européen comme maghrébin ${ }^{23}$.

Les éditions françaises de Mon Nerf et de Viscéral parues au Seuil ont un aspect plus «grand public». Elles émanent d'une maison d'édition connue qui ajoute de nombreux livres à son catalogue chaque année. La première de couverture a un aspect marketing et joue sur des codes de reconnaissance qui font consensus et sont susceptibles d'attirer le lecteur. L'illustration est vue comme le moment arrêté d'une histoire personnelle. La couverture peut intriguer afin d'inciter à la lecture, mais elle garde une dimension narrative, de témoignage. L'édition italienne, par contre, s'adresse à des lecteurs ouverts à une littérature plus expérimentale. En outre, la question des banlieues telle que la vit la France dans un contexte postcolonial est éloignée du cadre de référence du lecteur italien, tout comme

23. La photo a été proposée par Djaïdani lui-même : il s'agit d'un de ses amis, photographié en train de mettre ses bandes de boxe (information fournie par Ilaria Vitali, traductrice du roman en italien). 
la figure du «beur». Cela explique que l'éditeur n'ait pas utilisé ce motif, mais ait plutôt cherché à évoquer, par les connotations liées à l'image.

\section{Conclusion}

La première de couverture ajoute un cadre de référence à une traduction. Elle rend compte des options de l'éditeur et est destinée à un lectorat nécessairement différent, car de langue et de culture distinctes. De ce point de vue, elle est réinterprétation en fonction d'un autre contexte de lecture. Elle est un autre regard porté sur le texte, par un autre éditeur, pour un autre lecteur. Dans les romans que nous avons étudiés, les éditions en français cherchent à rendre proches les récits et, pour ce faire, tendent à personnaliser les premières de couverture. Les éditions traduites, quant à elles, élargissent le champ du regard, les unes en contextualisant dans une visée documentaire, les autres, plus expérimentales, en jouant sur le vague et l'inattendu - en mettant en valeur, finalement, le fait qu'il ne s'agit pas uniquement d'une littérature de la banlieue, sur la banlieue. Dans tous les cas, ce décentrement du regard nous semble résulter du fait que le proche qui peut être un argument marketing pour des éditeurs français l'est beaucoup moins dans un contexte plus éloigné. Il en est de même pour la recherche de valorisation d'un territoire stigmatisé.

La première de couverture a pour fonction d'attirer le lecteur, de lui donner envie de lire louvrage, mais aussi d'en figurer le contenu, dont elle cherche à reproduire également l'atmosphère. Elle est à lire comme un moment arrêté d'un scénario, tout en étant l'image d'une maison d'édition, d'une époque et d'une culture. Polyphonique, elle se doit de plaire au lecteur et permet à l'éditeur d'établir une connivence avec ce dernier. Ce pouvoir de l'image, sa richesse du point de vue de ses potentialités de communication, à la fois expressives, référentielles, poétiques et réceptives (ou conatives $)^{24}$ font que l'usage d'une couverture illustrée s'impose désormais aux éditeurs. Apparues avec les éditions de poche, les collections de prestige françaises ont longtemps affiché leur différence en mettant en avant le nom même de leurs collections : la Blanche de Gallimard, la Bleue du Mercure de France ou Cadre Rouge du Seuil. De nos jours, ces noms restent, mais la jaquette, amovible, devient couverture et remplit toutes les fonctions des

24. Nous reprenons ici les termes utilisés par Roman Jakobson (1963, p. 220). 
premières illustrées. La jaquette se fait en outre protection et écrin, renforçant le prestige de ce qu'elle recouvre. Ce n'est plus l'illustration désormais qui marque la différence, mais la présence de cette dernière. Les maisons d'édition françaises continuent cependant à promouvoir par l'image le nom de marque de leur collection. La couleur est reprise dans les titres ou les noms d'auteur figurant sur les jaquettes ou les couvertures, voire sur les bandeaux. Sur la jaquette de Boumkoeur de Rachid Djaidani, un cadre rouge barre le regard de l'auteur (voir l'illustration, p. 38). Ces éléments qui signent l'identité d'une Maison sont liés à l'histoire culturelle de l'édition, différente selon les pays. Et c'est aussi cette histoire que nous racontent les premières de couverture et que nous font lire, dans leurs spécificités, les différentes éditions des œuvres traduites. Mais nous sommes là dans ce qui ne se traduit pas.

\section{Références}

\section{Cuvres romanesques}

Begag, Azouz (1986). Le Gone du Chaâba. Paris, Seuil, coll. Points.

Begag, Azouz (1998). Le Gone du Chaâba. Paris, Seuil, coll. Cadre Rouge. $21 \mathrm{~cm}$.

Begag, Azouz (2005). Le Gone du Chaâba. Paris, Seuil, coll. Points. $18 \mathrm{~cm}$. [Photo Alain Dagbert/4 Lunes, affiche du film de Christophe Ruggia]

Begag, Azouz (2007). Shanty-town Kid. Trad. Naïma Wolf et Alec G. Hargreaves. Lincoln NE, University of Nebraska Press. $22 \mathrm{~cm}$. [Couverture A. Shahan. Photo Hulton Deutsch/Corbis]

Charef, Mehdi (1983). Le Thé au harem d'Archi Abmed. Paris, Mercure de France.

Charef, Mehdi (1988a). Le Thé au harem d'Archi Ahmed. Paris, Gallimard, coll. Folio. $18 \mathrm{~cm}$.

Charef, Mehdi (1988b). Le Thé au harem d'Archi Ahmed. Paris, Gallimard, coll. Folio. $18 \mathrm{~cm}$. [Photo Didier Lefevre/Vu.]

Charef, Mehdi (1989). Tea in the Harem. Trad. Ed Emery. Londres, Profile Books/Serpent's Tail. $20 \mathrm{~cm}$.

Djaïdani, Rachid (1999). Boumkoeur. Paris, Seuil, coll. Cadre Rouge. 19 cm. [Photo Christophe Boutin]

Djaïdani, Rachid (2004). Mon Nerf. Paris, Seuil, coll. Cadre Rouge. 19 cm. [Photo Siegfried]

Djaidani, Rachid (2005). Boumkoeur. Paris, Seuil, coll. Points. $18 \mathrm{~cm}$. [Photo Christophe Boutin]

Djaïdani, Rachid (2005). Mon Nerf. Paris, Seuil, coll. Points. $18 \mathrm{~cm}$. [Couverture Siegfried]

Djaïdani, Rachid (2007). Viscéral. Paris, Seuil, coll. Cadre Rouge. $21 \mathrm{~cm}$. [Photo Jeremy Stiester] 
Djaïdani, Rachid (2008). Viscéral. Paris, Seuil, coll. Points. 18 cm. [Photo Jeremy Stiester]

Djaidani, Rachid (2009).Viscerale. Un grido dalle banlieue. Trad.Ilaria Vitali. Rome, Giulio Perrone. $20 \mathrm{~cm}$. [Photo Rachid Djaïdani]

Djaïdani, Rachid (2011). Ritratto di un ragazzo da buttare alle ortiche. Trad. Ilaria Vitali. Rome, Giulio Perrone.

Guène, Faïza (2004). Kiffe kiffe demain. Paris, Hachette Littératures. $20 \mathrm{~cm}$. Guène, Faïza (2005). Kiffe kiffe demain. Paris, Librairie générale française, coll. Le livre de poche. $18 \mathrm{~cm}$. [Couverture Basso Cannarsa/Opale]

Guène, Faïza (2006). Kiffe Kiffe Tomorrow, Trad. Sarah Adams. New York, Houghton Mifflin Harcourt. $21 \mathrm{~cm}$. [Illustration Thomas Brodin/ Technikart]

Guène, Faïza (2010). Kiffe kiffe demain. Paris, Fayard.

Santaki, Rachid (2008). La petite cité dans la prairie. Lormont, Éditions Le Bord de l'Eau.

\section{Autres références}

Carpenter, Juliet et Christina Horvath, dir. (2015). Regards croisés sur la banlieue. Bruxelles, Peter Lang.

Childress, Clayton (2017). Under the Cover: The Creation, Production and Reception of a Novel. Princeton, Princeton University Press.

Corbis Historical (1961). «Algerian Shanty Town Near Paris». Disponible à <https://www.gettyimages.fr/license/613477186> [consulté le 30 juillet 2018].

Éditions Philippe Picquier (s.d.). Éditions Picquier. Littératures d'Asie. Disponible à <http://www.editions-picquier.com/> [consulté le 30 juillet 2018].

Elefante, Chiara (2015). «La littérature urbaine à l'épreuve de la traduction en italien. Une analyse socio-édito-traductologique». Repères-DoRiF, 8. Disponible à <http://dorif.it/ezine/ezine_articles.php?art_id=241> [consulté le 30 juillet 2018].

Fumigène (2007). «Qui fait la France?» Afrik.com. Disponible à <https:// www.afrik.com/qui-fait-la-france> [consulté le 30 juillet 2018].

Genette, Gérard (2002 [1987]). Seuils. Paris, Seuil.

Goudaillier, Jean-Pierre (1997). Comment tu tchatches! Dictionnaire du français contemporain des cités. Paris, Maisonneuve et Larose.

Goudaillier, Jean-Pierre (2002). «De l'argot traditionnel au français contemporain des cités ». La Linguistique, 38, 1, p. 5-24.

Goudaillier, Jean-Pierre (2007). «Français contemporain des cités (FCC) : langue en miroir, langue du refus de la société». Adolescence, 59, 1, p. $119-124$.

Goudaillier, Jean-Pierre (2015). «Culture "banlieues”, langue des “cités” et Internet». Hermès, 71, 1, p. 208-213.

Jakobson, Roman (1963). «Linguistique et poétique». In Essais de linguis- 
tique générale. Paris, Éditions de Minuit.

Manotti, Dominique (2007). «Roman noir». Le Mourvement social, 219220, p. 107-109.

Mauger, Gérard (2006). L'émeute de novembre 2005. Une révolte protopolitique. Bellecombe-en-Bauges, Éd. du Croquant.

Peirce, Charles S. (2002). Euvres I, Pragmatisme et pragmaticisme. Trad. Claudine Tiercelin et Pierre Thibaud. Paris, Éd. du Cerf.

Reuter, Yves (1981). «L'objet livre». Pratiques, 32, p. 105-113.

Turpin, Béatrice (2012). "Discours médiatiques et sémiotisation de lespace. La banlieue et ses grands ensembles». In B. Turpin, dir. Discours et sémiotisation de l'espace. Les représentations de la banlieue et de sa jeunesse. Paris, L'Harmattan, p. 103-121.

Turpin, Béatrice (2015). «Regards croisés sur la banlieue et ses grands ensembles». In J. Carpenter et C. Horvath, dir. Regards croisés sur la banlieue. Bruxelles, Peter Lang, p. 123-140.

Vitali, Ilaria (2009). «De la littérature beure à la littérature urbaine : le regard des "Intrangers"». Nouvelles études francophones, 24, 1, p. 172183.

Vitali, Ilaria (2012). «Une traduction "puissance trois" : Rachid Djaïdani et la langue des cités». Traduire, 226, p. 108-119.

Wiktionnaire (n.d.). «chaâba». Disponible à <https://fr.wiktionary.org/ wiki/cha\%C3\%A2ba> [consulté le 2 décembre 2018].

\author{
Béatrice Turpin \\ Laboratoire LT2D \\ Université de Cergy-Pontoise \\ Cergy-Pontoise, France \\ beatrice.turpin@u-cergy.fr
}

